\title{
Allelic database and accession divergence of a Brazilian mango collection based on microsatellite markers
}

\author{
I.C.N. dos Santos Ribeiro ${ }^{1}$, F.P. Lima Neto ${ }^{2}$ and C.A.F. Santos ${ }^{2}$ \\ ${ }^{1}$ Programa de Pós-Graduação em Recursos Genéticos Vegetais, \\ Universidade Estadual de Feira de Santana, Feira de Santana, BA, Brasil \\ ${ }^{2}$ Centro de Pesquisa Agropecuária do Trópico Semiárido, \\ Embrapa, Petrolina, PE, Brasil \\ Corresponding author: C.A.F. Santos \\ E-mail: casantos@cpatsa.embrapa.br
}

Genet. Mol. Res. 11 (4): 4564-4574 (2012)

Received February 28, 2012

Accepted June 13, 2012

Published October 9, 2012

DOI http://dx.doi.org/10.4238/2012.October.9.4

\begin{abstract}
Allelic patterns and genetic distances were examined in a collection of 103 foreign and Brazilian mango (Mangifera indica) accessions in order to develop a reference database to support cultivar protection and breeding programs. An UPGMA dendrogram was generated using Jaccard's coefficients from a distance matrix based on 50 alleles of 12 microsatellite loci. The base pair number was estimated by the method of inverse mobility. The cophenetic correlation was 0.8 . The accessions had a coefficient of similarity from 30 to $100 \%$, which reflects high genetic variability. Three groups were observed in the UPGMA dendrogram; the first group was formed predominantly by foreign accessions, the second group was formed by Brazilian accessions, and the Dashehari accession was isolated from the others. The 50 microsatellite alleles did not separate all 103 accessions, indicating that there are duplicates in this mango collection. These 12 microsatellites need to be validated in order to establish a reliable set to identify mango cultivars.
\end{abstract}

Key words: Mangifera indica; Variability; Dendrogram; Genotyping 


\section{INTRODUCTION}

Total world mango production is of the order of 26 million tons, and it is one of the most important fruits in the world, along with bananas, oranges, grapes, and apples. Countries such as India, China, Thailand, Mexico, Pakistan, and Indonesia are responsible for more than $75 \%$ of world mango production, with India constituting approximately $40 \%$ of this production (Viruel et al., 2005).

It is probable that mango cultivation started in India, where more than 1000 varieties have already been identified, resulting from selections within cross-pollinated populations. Traders spread cultivation from the center of origin and domestication to other tropical and subtropical regions. The Portuguese introduced the crop to West and East Africa and Brazil. From Brazil, it was probably taken to the Caribbean Islands, from where the Spanish introduced it to Mexico and the Philippines. In the 19th century, mango was introduced to Florida, USA, first from the Caribbean and later from India (Viruel et al., 2005).

DNA markers have been applied to mango principally to identify cultivars and the relationship between them (Krishna and Singh, 2007), probably with a single potential application in the selection of polyembrionic types assisted by random amplification of polymorphic DNA (RAPD) markers (López-Valenzuela et al., 1997). In addition to their utility for improvement and mapping, microsatellites have been the best markers for fingerprinting studies due to their high polymorphism, co-dominance, and reproducibility. Viruel et al. (2005), Duval et al. (2005), Honsho et al. (2005), and Schnell et al. (2006) developed and published around 65 microsatellites for mango.

Singh and Bhat (2009) analyzed 241 mango accessions from 15 different regions of India, identifying the existence of high variability among the accessions studied, as well as substantial gene flow among the accessions from different regions. Gálvez-López et al. (2009) found 2 specific groups of mango native to different Mexican states among 112 accessions from 16 different states analyzed with microsatellite markers and AFLP. Viruel et al. (2005), Duval et al. (2005), Olano et al. (2005), and Schnell et al. (2006) have reported other studies of mango diversity with microsatellites. In Brazil, studies have not yet been carried out with microsatellites in mango. Santos et al. (2008) reported studies with 157 AFLP markers in 104 mango accessions and the existence of high genetic variability among the accessions.

In spite of the availability of microsatellite markers, the minimum number of this type of marker has not yet been proposed for mango to help protect cultivars and settle commercial disputes. This et al. (2004) proposed 6 relevant microsatellite markers to reveal clonal polymorphism in grape cultivars. Leão et al. (2009) used this set of microsatellites to characterize a Brazilian grape collection.

The objectives of the present study were to establish allelic patterns and estimate genetic distances based on microsatellite markers for 103 mango accessions to generate a reference database to support cultivar protection and settle possible commercial disputes as well as to guide breeding programs and genetic resources of the species.

\section{MATERIAL AND METHODS}

\section{Plant material and DNA extraction}

Young healthy leaves were collected from 103 mango accessions of the Active Germ- 
plasm Bank (BAG) of Embrapa Tropical Semi-Arid (Table 1) and maintained in the Mandacaru Experimental Station, Juazeiro, BA. The CTAB method (Doyle and Doyle, 1990) was adopted with minor modifications: a) 6000 and 10,000 rpm for the first and second centrifugation rounds, respectively, and b) addition of $2 \% 2$-mercaptoethanol, and water bath incubation at $60^{\circ} \mathrm{C}$ for $30 \mathrm{~min}$ for all samples. After adding $30 \mu \mathrm{L}$ Tris-EDTA, the solution was treated with $10 \%$ RNase to remove co-extracted RNAs. Quantification and DNA integrity analyses were carried out on $0.8 \%$ agarose gel, stained with ethidium bromide. The DNA samples were diluted to $10 \mathrm{ng} / \mu \mathrm{L}$ and stored at $-20^{\circ} \mathrm{C}$.

\begin{tabular}{|c|c|c|c|}
\hline Accessions & Origin & Accessions & Origin \\
\hline Alfa & Brazil & Juazeiro VI & Brazil \\
\hline Alphonso & India & Lita & Brazil \\
\hline Amarelinha & Brazil & Keitt & EUA \\
\hline Ametista & Brazil & Kensington & Australia \\
\hline Amrapali & India & Kent & EUA \\
\hline Apple DCG & Thailand & Langra & India \\
\hline Ataulfo & México & M-13269 & EUA \\
\hline Beta & Brazil & Mallindi & India \\
\hline Black Java & Australia & Maçã & Brazil \\
\hline Bonita & Brazil & Mallika & India \\
\hline Bourbon & Brazil & Manga d'água & Brazil \\
\hline Brazil & Brazil & Manguito & Brazil \\
\hline Calmon & Brazil & Manilla & Filipinas \\
\hline Carabao & Filipinas & Manzanillo & México \\
\hline Carlotão & Brazil & Mastruz & Brazil \\
\hline Caxangá & Brazil & Maya & México \\
\hline Chené & South Africa & Momi-K & EUA \\
\hline China & Brazil & Mon Amon & Thailand \\
\hline Comprida Roxa & Brazil & Morais & Brazil \\
\hline Coração Magoado & Brazil & Nego não Chupa & Brazil \\
\hline CPR & Brazil & Néldica & South Africa \\
\hline Dama de Ouro & Brazil & Olour & India \\
\hline Da Porta & Brazil & Ômega & Brazil \\
\hline Dashehari & India & Palmer & EUA \\
\hline Duncan & EUA & Papo de Peru I & Brazil \\
\hline Edward & EUA & Parwin & EUA \\
\hline Eldon & EUA & Pêssego DPV & Brazil \\
\hline Espada & Brazil & Pingo de Ouro & Brazil \\
\hline Espada 35 & Brazil & Pingo de Ouro DPV & Brazil \\
\hline Espada Itaparica & Brazil & Primor Amoreira & Brazil \\
\hline Espada Manteiga & Brazil & Princesa & Brazil \\
\hline Espada Vermelha & Brazil & Roxa & Brazil \\
\hline Espada Ouro & Brazil & Recife & Brazil \\
\hline Extrema & Brazil & $\mathrm{R} 2 \mathrm{E} 2$ & Australia \\
\hline Favo de Mel & Brazil & Rosa & Brazil \\
\hline Florigon & EUA & Rosary & Brazil \\
\hline Foice & Brazil & Ruby & EUA \\
\hline Haden & EUA & Salitre & Brazil \\
\hline Haden $2 \mathrm{H}$ & Brazil & Sta Alexandrina & Brazil \\
\hline Haden Rosa & Brazil & Scuper Many & EUA \\
\hline Heidi & South Africa & Simmonds & EUA \\
\hline Hilda & Brazil & Smith & EUA \\
\hline Imperial I & Brazil & Surpresa & Brazil \\
\hline Ipuçaba & Brazil & Tommy Atkins & EUA \\
\hline Irwin & EUA & Torbet & EUA \\
\hline Itamaracá & Brazil & Tyler Premier & EUA \\
\hline Itiúba & Brazil & Ubá & Brazil \\
\hline Joa & South Africa & Umbu & Brazil \\
\hline Juazeiro II & Brazil & Winter & EUA \\
\hline Juazeiro III & Brazil & Van Dyke & EUA \\
\hline \multirow[t]{2}{*}{ Juazeiro IV } & Brazil & Zill & EUA \\
\hline & & 65 & EUA \\
\hline
\end{tabular}




\section{DNA PCRs and resolution on polyacrylamide gels}

Twenty-eight microsatellites published by Duval et al. (2005) and 15 others published by Schnell et al. (2005) were evaluated in 5 BAG accessions to select those with better polymorphic resolution on polyacrylamide gels. The PCR amplifications were carried out in a final volume of $20 \mu \mathrm{L}$ containing $20 \mathrm{ng}$ DNA, $0.2 \mu \mathrm{M}$ of each primer, $200 \mu \mathrm{M}$ of each dNTP, $1.5 \mathrm{mM} \mathrm{MgCl}_{2}, 1 \mathrm{X}$ PCR buffer, and $0.75 \mathrm{U}$ Taq DNA polymerase enzyme. The thermocycler was programmed for the amplifications as follows: an initial cycle of $94^{\circ} \mathrm{C}$ for $4 \mathrm{~min}$, followed by 32 cycles at $94^{\circ} \mathrm{C}$ for $45 \mathrm{~s}, 56^{\circ} \mathrm{C}$ for $60 \mathrm{~s}, 72^{\circ} \mathrm{C}$ for $60 \mathrm{~s}$, and a final cycle at $7^{\circ} \mathrm{C}$ for $4 \mathrm{~min}$.

Half of the volume of the denaturing buffer of $98 \%$ formamide (10 mM EDTA, $\mathrm{pH}$ $8.0,1 \mathrm{mg} / \mathrm{mL}$ xylene cyanol, and $1 \mathrm{mg} / \mathrm{mL}$ bromophenol blue) was added to the PCR, followed by complete denaturation at $94^{\circ} \mathrm{C}$ for $5 \mathrm{~min}$ in the thermocycler. Amplified PCR products were separated on $6 \%$ polyacrylamide gel for approximately $3 \mathrm{~h}$ with constant $40-\mathrm{W}$ power. The gels were stained with silver nitrate according to the procedure described by Creste et al. (2001). The 103 accessions were genotyped on 2 polyacrylamide gel plates, 1 plate with 56 accessions and the other with the remaining 47. On the first plate, at least 1 accession representing 1 allelic combination was identified, and was used as a reference allele on the second plate for each microsatellite.

\section{Microsatellite data analysis}

The microsatellite loci that presented easily interpreted polymorphisms were selected to genotype the 103 mango accessions. To construct the allelic pattern of each accession, the size estimate in base pairs (bp) for each allele was obtained by the inverse mobility method based on the regression of products of known size of the 50-bp molecular marker (Fermentas Inc., USA), applied in an extra well on the polyacrylamide gel.

The microsatellites were observed for the presence (1) versus absence ( 0 ) of alleles to construct a similarity matrix of Jaccard's index. A dendrogram for the distances of the cultivars was created by the UPGMA grouping method in the NTSYS program (Rohlf, 1989). The adjustment of the dendrogram phenogram was evaluated by cophenetic correlation. The frequency of the principal alleles, number of genotypes, gene diversity, heterozygosity, and polymorphic information content (PIC) per microsatellite were estimated with the PowerMarker program (Liu and Muse, 2005).

\section{RESULTS}

Easily identified polymorphic amplifications were obtained only in the mMiCIR001, mMiCIR003, mMiCIR010, mMiCIR027, mMiCIR028, mMiCIR030, and mMiCIR036 microsatellite loci developed by Duval et al. (2005), and the MiSHRS-1, MiSHRS-4, MiSHRS-29, and MiSHRS-32 microsatellite loci developed by Schnell et al. (2005). Fifty alleles were detected in the 11 microsatellites analyzed, in which the number of alleles per locus varied from 2 to 8 , with an average of 4 alleles per microsatellite in the 103 mango accessions genotyped. The largest number of genotypes and the highest gene diversity were observed with the mMiCIR030 microsatellite (Table 2). 


\begin{tabular}{|c|c|c|c|c|c|c|c|}
\hline Microsatellite & $\begin{array}{l}\text { Highest allelic } \\
\text { frequency }\end{array}$ & $\begin{array}{l}\text { Genotype } \\
\text { number }\end{array}$ & $\begin{array}{c}\text { No. of } \\
\text { accessions }\end{array}$ & $\begin{array}{l}\text { No. of } \\
\text { alleles }\end{array}$ & $\begin{array}{l}\text { Genetic } \\
\text { diversity }\end{array}$ & $\begin{array}{c}\text { Observed } \\
\text { heterozygosity }\end{array}$ & PIC \\
\hline MiSHRS-1 & 0.3155 & 16 & 103 & 7 & 0.7789 & 0.5146 & 0.7446 \\
\hline MiSHRS-4 & 0.5243 & 9 & 103 & 4 & 0.6348 & 0.6311 & 0.5816 \\
\hline MiSHRS-29 & 0.5243 & 12 & 103 & 5 & 0.6666 & 0.7476 & 0.6320 \\
\hline MiSHRS-32 & 0.5291 & 8 & 103 & 4 & 0.5773 & 0.2816 & 0.4956 \\
\hline mMiCIR030 & 0.3058 & 20 & 103 & 8 & 0.7864 & 0.8252 & 0.7543 \\
\hline mMiCIR001 & 0.9417 & 4 & 103 & 3 & 0.1102 & 0.0971 & 0.1051 \\
\hline mMiCIR003 & 0.6262 & 3 & 103 & 2 & 0.4681 & 0.4757 & 0.3586 \\
\hline mMiCIR010 & 0.7233 & 9 & 103 & 4 & 0.4495 & 0.4563 & 0.4204 \\
\hline mMiCIR027 & 0.8911 & 3 & 101 & 2 & 0.1941 & 0.1188 & 0.1753 \\
\hline mMiCIR028 & 0.7524 & 6 & 103 & 3 & 0.3990 & 0.3495 & 0.3591 \\
\hline mMiCIR036 & 0.8689 & 6 & 103 & 4 & 0.2391 & 0.2330 & 0.2302 \\
\hline Average & 0.6200 & 9 & 103 & 4 & 0.4948 & 0.4500 & 0.4516 \\
\hline
\end{tabular}

$\mathrm{PIC}=$ polymorphic information content.

The averaged observed heterozygosity and PIC of the 11 microsatellite loci was 0.45 and 0.45 , respectively, and the MiSHRS- 1 and mMiCIR030 loci presented the highest values (Table 2). These two parameters represent the existence of variability because each diploid individual can have up to two alleles per locus (Weir, 1996), in which variability is greater with a higher frequency of heterozygotes and PIC, and the microsatellites that fit this situation are considered the most suitable for diversity studies.

The cophenetic correlation was 0.8 , which indicated that the produced dendrogram (Figure 1) presents some inconsistencies in the grouping of the 103 mango accessions with the 50 alleles of the 11 microsatellite loci. This low cophenetic correlation may have been due to the presence of ties in the matrix of similarity, as commented on by Santos et al. (2010) in onion.

The accessions presented a similarity coefficient between 30 and $100 \%$, which reflects the high genetic variability of the collection of mango germplasm studied (Figure 1). Three groups were observed in the dendrogram (Figure 1): group I ranged from Scuper Many to Beta, group II ranged from Rosari to Espada Manteiga, and group III was formed exclusively by Dashehari. Group I was formed predominantly by foreign accessions or the results of crossings between these accessions, such as the Beta and Alfa accession, and by 6 Brazilian accessions as well: Maça, Surpresa, Juazeiro II, Umbu, Ipuçaba, and Pingo de Ouro DPV. Group II was formed by accessions that owe their origin to long adaptation following the introduction of mango to Brazil. Other studies should be carried out to elucidate the isolated position of the Dashehari accession that originated from India, as well as the 6 accessions of notably Brazilian origin in the group of accessions introduced recently from other countries.

The 50 alleles of the 11 SSR loci were not sufficient to separate all 103 mango accessions, suggesting the existence of duplicates in the collection for the following accessions: Haden Rosa and Haden, Kensington and Black Java, Nego não Chupa and Bourbon, Espada and Espada35, Papo de Peru I and Salitre and CPR, and finally, Santa Alexandrina and Itamaracá (Table 3 and Figure 1). Santos et al. (2010) differentiated 44 onion accessions with 13 microsatellite loci; Priolli et al. (2002) differentiated 184 soybean cultivars applying only 12 microsatellite SSR markers. Leão et al. (2009) divided a collection of 221 grape accessions into 4 groups using 7 microsatellite loci as a reference set. 


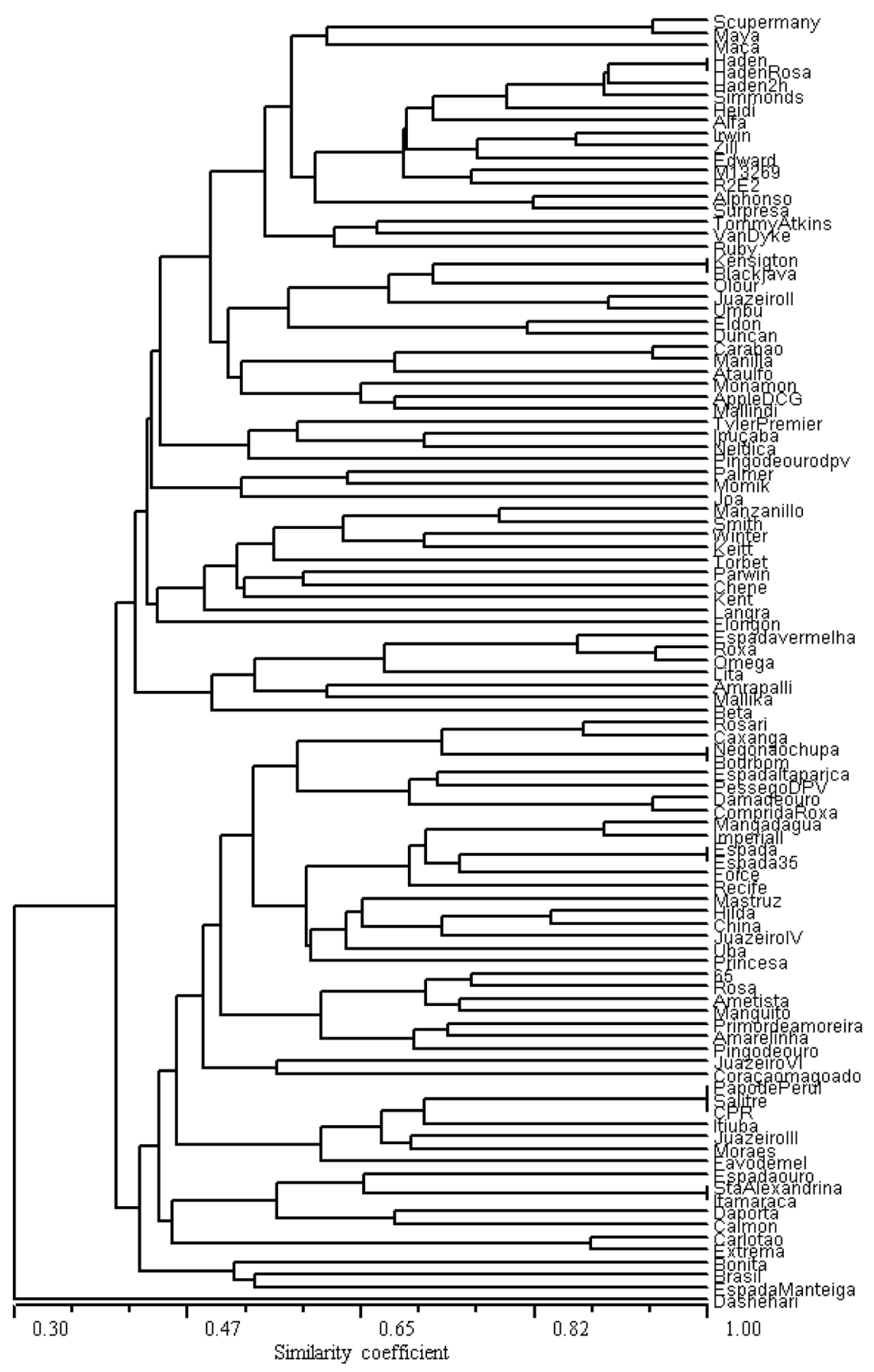

Figure 1. UPGMA dendrogram of Jacquard's coefficient of similarity of 103 mango accessions genotyped for 50 alleles of 12 microsatellite loci. Cophenetic correlation $=0.8$. 


\begin{tabular}{|c|c|c|c|c|c|c|c|c|c|c|c|}
\hline \multirow[t]{2}{*}{ Accession } & \multicolumn{4}{|c|}{ MiSHRS locus } & \multicolumn{7}{|c|}{ mMiCIR locus } \\
\hline & 1 & 4 & 29 & 32 & 030 & 001 & 003 & 010 & 027 & 028 & 036 \\
\hline 65 & $492 / 539$ & $197 / 197$ & $330 / 356$ & $554 / 554$ & $295 / 301$ & $412 / 412$ & $773 / 758$ & $749 / 783$ & $218 / 243$ & $694 / 694$ & $751 / 751$ \\
\hline Alfa & $492 / 548$ & $197 / 212$ & $356 / 362$ & $581 / 581$ & $308 / 308$ & $412 / 412$ & $773 / 758$ & $776 / 783$ & $243 / 243$ & $659 / 694$ & $693 / 751$ \\
\hline Alphonso & $548 / 548$ & $197 / 197$ & $343 / 356$ & $595 / 595$ & $256 / 308$ & $412 / 412$ & $773 / 758$ & $783 / 783$ & $243 / 243$ & $659 / 659$ & $751 / 751$ \\
\hline Amarelinha & $492 / 492$ & $197 / 197$ & $343 / 356$ & $554 / 554$ & $295 / 301$ & $412 / 412$ & $773 / 758$ & $749 / 783$ & $218 / 243$ & $659 / 659$ & $751 / 751$ \\
\hline Ametista & $492 / 492$ & $197 / 197$ & $330 / 375$ & $554 / 554$ & $295 / 301$ & $412 / 412$ & $773 / 758$ & $783 / 783$ & $218 / 243$ & $659 / 694$ & $751 / 751$ \\
\hline Amrapalli & $492 / 548$ & $204 / 212$ & $356 / 362$ & $554 / 595$ & $301 / 318$ & $412 / 412$ & $773 / 758$ & $783 / 783$ & $243 / 243$ & $659 / 694$ & $693 / 751$ \\
\hline Apple DCG & $539 / 548$ & $197 / 197$ & $356 / 356$ & $581 / 581$ & $318 / 318$ & $412 / 428$ & 773/773 & $755 / 783$ & $243 / 243$ & $659 / 659$ & $751 / 751$ \\
\hline Ataulfo & $384 / 539$ & $197 / 204$ & $356 / 356$ & $581 / 581$ & $256 / 328$ & $412 / 412$ & $773 / 773$ & $755 / 783$ & $243 / 243$ & $694 / 694$ & $751 / 751$ \\
\hline Beta & $384 / 539$ & $192 / 204$ & $356 / 356$ & $595 / 595$ & $295 / 328$ & $412 / 412$ & $773 / 758$ & $783 / 783$ & $243 / 243$ & $659 / 694$ & $693 / 751$ \\
\hline Black Java & $548 / 548$ & $197 / 197$ & $330 / 356$ & $581 / 581$ & $295 / 328$ & $412 / 412$ & $773 / 773$ & $783 / 783$ & $243 / 243$ & $659 / 659$ & $728 / 751$ \\
\hline Bonita & $492 / 492$ & $192 / 197$ & $356 / 375$ & $554 / 554$ & $301 / 328$ & $412 / 412$ & $773 / 758$ & $783 / 783$ & $218 / 218$ & $659 / 694$ & $751 / 751$ \\
\hline Bourbom & $510 / 539$ & $197 / 204$ & $343 / 356$ & $554 / 554$ & $295 / 328$ & $412 / 412$ & 773/773 & 783/783 & $243 / 243$ & $659 / 659$ & $751 / 751$ \\
\hline Brazil & $492 / 510$ & $197 / 204$ & $343 / 356$ & $554 / 581$ & $301 / 308$ & $412 / 412$ & 773/773 & $783 / 783$ & $218 / 218$ & $659 / 694$ & $714 / 751$ \\
\hline Calmon & $510 / 510$ & $204 / 204$ & $330 / 330$ & $554 / 581$ & $308 / 311$ & $412 / 412$ & $773 / 773$ & $749 / 783$ & $243 / 243$ & $659 / 659$ & $751 / 751$ \\
\hline Carabao & $539 / 548$ & 197/197 & $343 / 356$ & $595 / 595$ & $256 / 328$ & $412 / 412$ & 773/773 & $755 / 783$ & $243 / 243$ & $659 / 694$ & $751 / 751$ \\
\hline Carlotão & $510 / 548$ & $197 / 197$ & $375 / 375$ & $554 / 581$ & $295 / 308$ & $412 / 428$ & $773 / 773$ & $783 / 783$ & $243 / 243$ & $659 / 659$ & $751 / 751$ \\
\hline Caxangá & $510 / 510$ & $197 / 204$ & $343 / 356$ & $554 / 554$ & $295 / 295$ & $412 / 412$ & $773 / 773$ & $783 / 783$ & $243 / 243$ & $659 / 659$ & $751 / 751$ \\
\hline Chené & $510 / 548$ & $204 / 212$ & $356 / 356$ & $554 / 581$ & $301 / 328$ & $412 / 423$ & $758 / 758$ & $776 / 783$ & $243 / 243$ & $659 / 694$ & $751 / 751$ \\
\hline China & $510 / 539$ & $197 / 204$ & $343 / 356$ & $581 / 581$ & 295/301 & $412 / 412$ & $773 / 773$ & $755 / 783$ & $243 / 243$ & $659 / 694$ & $751 / 751$ \\
\hline C. Roxa & $539 / 539$ & $204 / 204$ & $356 / 375$ & $554 / 554$ & $295 / 301$ & $412 / 412$ & $773 / 758$ & $749 / 783$ & $243 / 243$ & $659 / 659$ & $751 / 751$ \\
\hline C. Magoado & $548 / 548$ & $197 / 204$ & $330 / 356$ & $554 / 640$ & $295 / 301$ & $412 / 412$ & $773 / 773$ & $755 / 783$ & $218 / 243$ & $659 / 694$ & $714 / 751$ \\
\hline CPR & $384 / 548$ & $197 / 204$ & $356 / 375$ & $581 / 581$ & $295 / 328$ & $412 / 412$ & $773 / 758$ & $749 / 783$ & $243 / 243$ & $659 / 659$ & $751 / 751$ \\
\hline Da Porta & $510 / 539$ & $204 / 204$ & $330 / 375$ & $554 / 554$ & $301 / 308$ & $412 / 412$ & $773 / 758$ & $749 / 783$ & $243 / 243$ & $659 / 659$ & $714 / 751$ \\
\hline Dama Ouro & $492 / 539$ & $204 / 204$ & $356 / 375$ & $554 / 554$ & $295 / 301$ & $412 / 412$ & $773 / 758$ & $749 / 783$ & $243 / 243$ & $659 / 659$ & $751 / 751$ \\
\hline Dashehari & $529 / 539$ & $212 / 212$ & $343 / 356$ & $595 / 595$ & $308 / 308$ & $412 / 412$ & $758 / 758$ & $783 / 783$ & $243 / 243$ & $670 / 670$ & $728 / 751$ \\
\hline Duncan & $539 / 548$ & $197 / 204$ & $330 / 356$ & $581 / 581$ & $295 / 318$ & $412 / 412$ & $758 / 758$ & $749 / 783$ & $243 / 243$ & $659 / 670$ & $714 / 751$ \\
\hline Edward & $548 / 548$ & $197 / 197$ & $356 / 362$ & $581 / 581$ & $308 / 308$ & $412 / 412$ & $773 / 758$ & $755 / 783$ & $243 / 243$ & $659 / 659$ & $751 / 751$ \\
\hline Eldon & $539 / 548$ & $197 / 204$ & $330 / 356$ & $581 / 581$ & $295 / 318$ & $412 / 412$ & $758 / 758$ & $755 / 783$ & $243 / 243$ & $659 / 670$ & $728 / 751$ \\
\hline Espada & $384 / 510$ & $197 / 204$ & $330 / 356$ & $554 / 581$ & $295 / 301$ & $412 / 412$ & $773 / 773$ & $749 / 783$ & $243 / 243$ & $659 / 659$ & $751 / 751$ \\
\hline Espada 35 & $384 / 510$ & $197 / 204$ & $330 / 356$ & $554 / 581$ & $295 / 301$ & $412 / 412$ & $773 / 773$ & $749 / 783$ & $243 / 243$ & $659 / 659$ & $751 / 751$ \\
\hline Esp. Itaparica & $510 / 539$ & $197 / 204$ & $330 / 375$ & $554 / 554$ & $295 / 301$ & $412 / 412$ & 773/758 & $749 / 783$ & $243 / 243$ & $659 / 659$ & $751 / 751$ \\
\hline Esp. Manteiga & $492 / 510$ & $204 / 212$ & $356 / 356$ & $554 / 554$ & $301 / 308$ & $412 / 412$ & $773 / 758$ & $783 / 783$ & $243 / 243$ & $670 / 694$ & $751 / 751$ \\
\hline Espada Ouro & $510 / 548$ & $197 / 204$ & $330 / 375$ & $554 / 554$ & $301 / 328$ & $412 / 412$ & $773 / 758$ & $749 / 783$ & $218 / 243$ & $659 / 694$ & $751 / 751$ \\
\hline Esp. Vermelha & $492 / 548$ & $197 / 204$ & $356 / 356$ & $581 / 581$ & $308 / 308$ & $412 / 412$ & $758 / 758$ & $783 / 783$ & $243 / 243$ & $659 / 694$ & $693 / 751$ \\
\hline Extrema & $510 / 548$ & $197 / 197$ & $362 / 375$ & $554 / 581$ & 295/295 & $412 / 428$ & 773/773 & $783 / 783$ & - & $659 / 659$ & $714 / 751$ \\
\hline Favo de Mel & $492 / 510$ & $204 / 204$ & $356 / 356$ & $581 / 581$ & $295 / 301$ & $412 / 412$ & 773/758 & $783 / 783$ & $243 / 243$ & $659 / 659$ & $751 / 751$ \\
\hline Florigon & $539 / 539$ & $192 / 212$ & $343 / 356$ & $581 / 581$ & 295/295 & $412 / 412$ & 773/773 & $776 / 783$ & $243 / 243$ & $670 / 670$ & $751 / 751$ \\
\hline Foice & $384 / 384$ & $197 / 204$ & $343 / 356$ & $554 / 581$ & $295 / 301$ & $412 / 412$ & 773/773 & $749 / 749$ & $243 / 243$ & $659 / 659$ & $751 / 751$ \\
\hline Haden & $548 / 548$ & $197 / 212$ & $356 / 362$ & $581 / 581$ & $295 / 308$ & $412 / 412$ & $773 / 758$ & $776 / 783$ & $243 / 243$ & $659 / 670$ & $751 / 751$ \\
\hline Haden $2 \mathrm{H}$ & $539 / 548$ & $197 / 212$ & $356 / 362$ & $581 / 581$ & $295 / 308$ & $412 / 412$ & $773 / 758$ & $776 / 783$ & $243 / 243$ & $659 / 659$ & $751 / 751$ \\
\hline Haden Rosa & $548 / 548$ & $197 / 212$ & $356 / 362$ & $581 / 581$ & $295 / 308$ & $412 / 412$ & $773 / 758$ & $776 / 783$ & $243 / 243$ & $659 / 670$ & $751 / 751$ \\
\hline Heidi & $548 / 548$ & $197 / 212$ & $356 / 356$ & $581 / 581$ & & $412 / 412$ & $773 / 758$ & $776 / 783$ & $243 / 243$ & $659 / 659$ & $751 / 751$ \\
\hline Hilda & $510 / 510$ & $197 / 204$ & $343 / 356$ & $581 / 581$ & $295 / 295$ & $412 / 412$ & 773/773 & $755 / 783$ & $243 / 243$ & $659 / 659$ & $751 / 751$ \\
\hline Imperial I & $384 / 510$ & $197 / 197$ & $343 / 356$ & $554 / 581$ & $295 / 328$ & $412 / 412$ & 773/773 & $749 / 783$ & $243 / 243$ & $659 / 659$ & $751 / 751$ \\
\hline Ipuçaba & $548 / 548$ & $192 / 197$ & $343 / 356$ & $554 / 581$ & $295 / 328$ & $412 / 412$ & 773/773 & $749 / 749$ & $218 / 218$ & $659 / 659$ & $751 / 751$ \\
\hline Irwin & $548 / 548$ & $197 / 197$ & $343 / 356$ & $581 / 581$ & $308 / 311$ & $412 / 412$ & $773 / 758$ & $783 / 783$ & $243 / 243$ & $659 / 659$ & $751 / 751$ \\
\hline Itamaracá & $510 / 548$ & $192 / 197$ & $330 / 375$ & $554 / 554$ & & $412 / 428$ & $773 / 758$ & $783 / 783$ & $243 / 243$ & $659 / 659$ & $751 / 751$ \\
\hline Itiúba & $384 / 384$ & $192 / 204$ & $356 / 375$ & $581 / 581$ & $301 / 328$ & $412 / 412$ & $773 / 758$ & $783 / 783$ & $243 / 243$ & $659 / 659$ & $751 / 751$ \\
\hline Joa & $492 / 492$ & $204 / 212$ & $356 / 362$ & $581 / 640$ & $308 / 328$ & $412 / 412$ & $773 / 758$ & $749 / 776$ & $218 / 243$ & $659 / 694$ & $714 / 751$ \\
\hline Juazeiro II & $539 / 548$ & $197 / 204$ & $330 / 356$ & $554 / 554$ & $295 / 328$ & $412 / 412$ & 773/773 & $783 / 783$ & $218 / 243$ & $659 / 659$ & $728 / 751$ \\
\hline Juazeiro III & $384 / 548$ & $197 / 204$ & $356 / 375$ & $581 / 581$ & $301 / 308$ & $412 / 412$ & $773 / 758$ & $783 / 783$ & $243 / 243$ & $659 / 659$ & $751 / 751$ \\
\hline Juazeiro IV & $510 / 510$ & $197 / 204$ & $356 / 375$ & $554 / 554$ & $295 / 301$ & $412 / 412$ & $773 / 773$ & $755 / 783$ & $243 / 243$ & $659 / 659$ & $751 / 751$ \\
\hline Juazeiro VI & $510 / 510$ & $204 / 204$ & $330 / 375$ & $554 / 554$ & $295 / 328$ & $412 / 412$ & $773 / 773$ & $755 / 783$ & $243 / 243$ & $659 / 694$ & $751 / 751$ \\
\hline Keitt & $510 / 548$ & $192 / 212$ & $356 / 362$ & $554 / 581$ & $295 / 328$ & $412 / 412$ & $773 / 758$ & $776 / 783$ & $243 / 243$ & $659 / 659$ & $751 / 751$ \\
\hline Kensigton & $548 / 548$ & $197 / 197$ & $330 / 356$ & $581 / 581$ & $295 / 328$ & $412 / 412$ & $773 / 773$ & $783 / 783$ & $243 / 243$ & $659 / 659$ & $728 / 751$ \\
\hline Kent & $510 / 510$ & $192 / 197$ & $356 / 356$ & $554 / 581$ & $308 / 328$ & $412 / 412$ & $758 / 758$ & $776 / 776$ & $243 / 243$ & $659 / 694$ & $751 / 751$ \\
\hline Langra & $384 / 384$ & $192 / 212$ & $356 / 375$ & $554 / 554$ & $256 / 328$ & $412 / 412$ & $773 / 758$ & 783/783 & $243 / 243$ & $659 / 659$ & $751 / 751$ \\
\hline Lita & $539 / 539$ & $197 / 204$ & $356 / 356$ & $581 / 581$ & $308 / 328$ & $412 / 412$ & $758 / 758$ & 783/783 & $243 / 243$ & $659 / 694$ & $693 / 751$ \\
\hline M-13269 & $548 / 548$ & $192 / 197$ & $343 / 356$ & $581 / 581$ & $292 / 308$ & $412 / 412$ & $773 / 758$ & $776 / 783$ & $243 / 243$ & $659 / 659$ & $751 / 751$ \\
\hline
\end{tabular}

Continued on next page 


\begin{tabular}{|c|c|c|c|c|c|c|c|c|c|c|c|}
\hline \multirow[t]{2}{*}{ Accession } & \multicolumn{4}{|c|}{ MiSHRS locus } & \multicolumn{7}{|c|}{ mMiCIR locus } \\
\hline & 1 & 4 & 29 & 32 & 030 & 001 & 003 & 010 & 027 & 028 & 036 \\
\hline Maçã & $510 / 510$ & $197 / 197$ & $356 / 362$ & $581 / 581$ & $308 / 328$ & $412 / 412$ & $773 / 773$ & $783 / 783$ & - & $659 / 659$ & $714 / 751$ \\
\hline Mallika & $492 / 539$ & $204 / 212$ & $356 / 362$ & $581 / 595$ & $301 / 328$ & $412 / 412$ & $773 / 758$ & $783 / 783$ & $243 / 243$ & $659 / 670$ & $693 / 728$ \\
\hline Mallindi & $539 / 539$ & $197 / 197$ & $356 / 356$ & $581 / 581$ & $318 / 328$ & $412 / 428$ & $773 / 758$ & $783 / 783$ & $243 / 243$ & $659 / 694$ & $751 / 751$ \\
\hline Manga d'água & $384 / 510$ & $197 / 197$ & $343 / 356$ & $554 / 581$ & $295 / 328$ & $412 / 428$ & $773 / 773$ & $749 / 783$ & $243 / 243$ & $659 / 659$ & $751 / 751$ \\
\hline Manguito & $492 / 548$ & $197 / 197$ & $330 / 330$ & $554 / 554$ & $295 / 301$ & $412 / 412$ & $773 / 758$ & $783 / 783$ & $218 / 243$ & $659 / 694$ & $751 / 751$ \\
\hline Manilla & $539 / 548$ & $197 / 197$ & $356 / 356$ & $595 / 595$ & $256 / 328$ & $412 / 412$ & $773 / 773$ & $755 / 783$ & $243 / 243$ & $659 / 694$ & $751 / 751$ \\
\hline Manzanillo & $492 / 492$ & $192 / 212$ & $356 / 362$ & $581 / 581$ & $295 / 328$ & $412 / 412$ & $758 / 758$ & $776 / 783$ & $243 / 243$ & $670 / 694$ & $751 / 751$ \\
\hline Mastruz & $510 / 510$ & $197 / 204$ & $330 / 356$ & $554 / 581$ & $301 / 301$ & $412 / 412$ & $773 / 773$ & $783 / 783$ & $218 / 243$ & $659 / 659$ & $751 / 751$ \\
\hline Maya & $384 / 548$ & $192 / 197$ & $356 / 356$ & $581 / 640$ & $308 / 328$ & $412 / 412$ & $773 / 773$ & $783 / 783$ & $243 / 243$ & $659 / 659$ & $751 / 751$ \\
\hline Momi-K & $492 / 492$ & $197 / 197$ & $343 / 362$ & $581 / 581$ & $308 / 328$ & $412 / 412$ & $773 / 758$ & $783 / 783$ & $243 / 243$ & $659 / 659$ & $728 / 728$ \\
\hline Mon Amon & $539 / 600$ & $197 / 212$ & $356 / 356$ & $581 / 581$ & $318 / 318$ & $412 / 428$ & $758 / 758$ & $783 / 783$ & $243 / 243$ & $659 / 659$ & $693 / 751$ \\
\hline Morais & $384 / 384$ & $204 / 204$ & $356 / 356$ & $581 / 581$ & $295 / 301$ & $412 / 412$ & $773 / 758$ & $749 / 783$ & $243 / 243$ & $659 / 659$ & $751 / 751$ \\
\hline Néldica & $548 / 548$ & $197 / 212$ & $343 / 356$ & $554 / 581$ & $295 / 328$ & $412 / 428$ & $773 / 773$ & $783 / 783$ & $218 / 218$ & $659 / 694$ & $751 / 751$ \\
\hline Nego não Chupa & $510 / 539$ & $197 / 204$ & $343 / 356$ & $554 / 554$ & $295 / 328$ & $412 / 412$ & $773 / 773$ & $783 / 783$ & $243 / 243$ & $659 / 659$ & $751 / 751$ \\
\hline Olour & $539 / 548$ & $197 / 197$ & $356 / 356$ & $581 / 581$ & $295 / 295$ & $412 / 412$ & $773 / 773$ & $783 / 783$ & $243 / 243$ & $659 / 694$ & $728 / 751$ \\
\hline Ômega & $492 / 548$ & $197 / 204$ & $356 / 362$ & $581 / 595$ & $308 / 308$ & $412 / 412$ & $758 / 758$ & $783 / 783$ & $243 / 243$ & $659 / 659$ & $693 / 751$ \\
\hline Palmer & $492 / 492$ & $197 / 204$ & $330 / 362$ & $554 / 581$ & $308 / 328$ & $412 / 412$ & $773 / 758$ & $776 / 783$ & $243 / 243$ & $659 / 694$ & $751 / 751$ \\
\hline Papo Peru I & $384 / 548$ & $197 / 204$ & $356 / 375$ & $581 / 581$ & $295 / 328$ & $412 / 412$ & $773 / 758$ & $749 / 783$ & $243 / 243$ & $659 / 659$ & $751 / 751$ \\
\hline Parwin & $492 / 492$ & $192 / 212$ & $330 / 356$ & $554 / 581$ & $301 / 328$ & $412 / 412$ & $758 / 758$ & $783 / 783$ & $243 / 243$ & $659 / 659$ & $751 / 751$ \\
\hline Pessego DPV & $510 / 539$ & $197 / 204$ & $343 / 343$ & $554 / 554$ & $295 / 301$ & $412 / 412$ & $773 / 758$ & $749 / 783$ & $218 / 243$ & $659 / 659$ & $751 / 751$ \\
\hline Pingo de Ouro & $539 / 539$ & $197 / 197$ & $343 / 356$ & $554 / 554$ & $295 / 308$ & $412 / 412$ & $773 / 758$ & $783 / 783$ & $243 / 243$ & $659 / 659$ & $751 / 751$ \\
\hline Pingo O. DPV & $510 / 548$ & $192 / 204$ & $343 / 356$ & $554 / 581$ & $328 / 328$ & $428 / 428$ & $773 / 773$ & $783 / 783$ & $243 / 243$ & $659 / 659$ & $751 / 751$ \\
\hline Pri. Amoreira & $492 / 539$ & $197 / 197$ & $330 / 356$ & $554 / 554$ & $295 / 301$ & $412 / 412$ & $773 / 758$ & $783 / 783$ & $243 / 243$ & $659 / 659$ & $751 / 751$ \\
\hline Princesa & $510 / 510$ & $197 / 197$ & $343 / 362$ & $554 / 554$ & $295 / 301$ & $412 / 412$ & $773 / 773$ & $749 / 783$ & $243 / 243$ & $659 / 659$ & $751 / 751$ \\
\hline R2E2 & $510 / 548$ & $192 / 197$ & $356 / 356$ & $581 / 581$ & $295 / 308$ & $412 / 412$ & $773 / 758$ & $776 / 783$ & $243 / 243$ & $659 / 694$ & $751 / 751$ \\
\hline Recife & $510 / 510$ & $197 / 204$ & $343 / 356$ & $554 / 581$ & $295 / 308$ & $412 / 412$ & $773 / 773$ & $749 / 783$ & $243 / 243$ & $659 / 694$ & $751 / 751$ \\
\hline Rosa & $492 / 510$ & $197 / 197$ & $330 / 330$ & $554 / 554$ & $295 / 301$ & $412 / 412$ & $773 / 773$ & $749 / 783$ & $218 / 243$ & $659 / 694$ & $751 / 751$ \\
\hline Rosary & $510 / 510$ & $197 / 204$ & $330 / 356$ & $554 / 554$ & $295 / 295$ & $412 / 412$ & $773 / 773$ & $783 / 783$ & $243 / 243$ & $659 / 659$ & $751 / 751$ \\
\hline Roxa & $492 / 548$ & $197 / 204$ & $356 / 362$ & $581 / 595$ & $308 / 308$ & $412 / 412$ & $758 / 758$ & $783 / 783$ & $243 / 243$ & $659 / 694$ & $693 / 751$ \\
\hline Ruby & $548 / 548$ & $197 / 212$ & $343 / 356$ & $581 / 640$ & $295 / 311$ & $412 / 412$ & $773 / 758$ & $783 / 783$ & $243 / 243$ & $670 / 694$ & $751 / 751$ \\
\hline Salitre & $384 / 548$ & $197 / 204$ & $356 / 375$ & $581 / 581$ & $295 / 328$ & $412 / 412$ & $773 / 758$ & $749 / 783$ & $243 / 243$ & $659 / 659$ & $751 / 751$ \\
\hline Scuper Many & $384 / 548$ & $192 / 197$ & $343 / 356$ & $581 / 640$ & $308 / 308$ & $412 / 412$ & $773 / 773$ & $783 / 783$ & $243 / 243$ & $659 / 659$ & $751 / 751$ \\
\hline Simmonds & $548 / 548$ & $197 / 212$ & $356 / 362$ & $581 / 581$ & $295 / 308$ & $412 / 412$ & $773 / 758$ & $783 / 783$ & $243 / 243$ & $659 / 659$ & $751 / 751$ \\
\hline Smith & $492 / 492$ & $192 / 212$ & $356 / 356$ & $581 / 581$ & $295 / 328$ & $412 / 412$ & $773 / 758$ & $783 / 783$ & $243 / 243$ & $670 / 670$ & $751 / 751$ \\
\hline SAlexandrina & $510 / 548$ & $192 / 197$ & $330 / 375$ & $554 / 554$ & $308 / 328$ & $412 / 428$ & $773 / 758$ & $783 / 783$ & $243 / 243$ & $659 / 659$ & $751 / 751$ \\
\hline Surpresa & $548 / 548$ & $197 / 197$ & $356 / 356$ & $595 / 595$ & $301 / 256$ & $412 / 412$ & $773 / 758$ & $783 / 783$ & $243 / 243$ & $659 / 659$ & $751 / 751$ \\
\hline T. Atkins & $548 / 548$ & $197 / 197$ & $330 / 356$ & $581 / 581$ & $308 / 311$ & $412 / 412$ & $773 / 758$ & $783 / 783$ & $243 / 243$ & $670 / 694$ & $693 / 751$ \\
\hline Torbet & $384 / 384$ & $212 / 212$ & $330 / 356$ & $581 / 581$ & $295 / 328$ & $412 / 412$ & $758 / 758$ & $755 / 776$ & $243 / 243$ & $659 / 659$ & $751 / 751$ \\
\hline Tyler Premier & $510 / 510$ & $192 / 197$ & $343 / 356$ & $581 / 581$ & $318 / 328$ & $412 / 412$ & $773 / 773$ & $755 / 755$ & $218 / 218$ & $659 / 659$ & $751 / 751$ \\
\hline Ubá & $510 / 510$ & $197 / 197$ & $356 / 375$ & $554 / 581$ & $295 / 301$ & $412 / 412$ & $773 / 773$ & $783 / 783$ & $243 / 243$ & $659 / 659$ & $751 / 751$ \\
\hline Umbu & $539 / 548$ & $197 / 204$ & $343 / 356$ & $554 / 554$ & $295 / 328$ & $412 / 412$ & $773 / 773$ & $783 / 783$ & $218 / 243$ & $659 / 659$ & $728 / 751$ \\
\hline Van Dyke & $539 / 548$ & $197 / 197$ & $356 / 362$ & $581 / 581$ & $308 / 311$ & $412 / 412$ & $758 / 758$ & $776 / 783$ & $243 / 243$ & $670 / 694$ & $751 / 751$ \\
\hline Winter & $384 / 384$ & $192 / 212$ & $356 / 356$ & $581 / 581$ & $295 / 328$ & $412 / 412$ & $773 / 758$ & $783 / 783$ & $243 / 243$ & $659 / 659$ & $751 / 751$ \\
\hline Zill & $548 / 548$ & $197 / 197$ & $356 / 356$ & $581 / 581$ & $308 / 308$ & $412 / 412$ & $773 / 758$ & $783 / 783$ & $243 / 243$ & $659 / 659$ & $751 / 751$ \\
\hline
\end{tabular}

\section{DISCUSSION}

In some situations, the SSR molecular markers can present additional information when morphological descriptors are insufficient to distinguish cultivars of a species with a narrow genetic base (Priolli et al., 2002). Jakse et al. (2005) suggested that additional markers should be used to reveal polymorphisms in situations in which it was not possible to distinguish accessions of a given species with a set of predetermined DNA markers. In addition to those tested in this study, additional microsatellites, or even adjustments to the annealing temperature of the PCR protocol of some of the microsatellites tested here, can be obtained in Viruel et al. (2005). 
The Carabao and Manilla accessions were considered genetically identical by Santos et al. (2008) because they had 97\% similarity when analyzed with the AFLP marker, and also by López-Valenzuela et al. (1997) when analyzed with RAPD. Although they presented high similarity in the present study (96\%), these accessions should not be considered identical because the MiSHRS-29 microsatellite differentiated them (Table 3).

The size of the alleles varied from 192 bp in MiSHRS-4 to 758 bp in mMiCIR010 (Table 3), presenting some inconsistency with the size reported by Duval et al. (2005) and Schnell et al. (2005). These discrepancies could be attributed to the method used to estimate fragment size in the present study or the presence of different alleles, since microsatellite markers are multiallelic. In spite of the expected approximate 500-bp size on polyacrylamide gel, Santos and Simon (2004) reported fragment size ranging from 54 to 700 nucleotides when analyzing AFLP amplicons with the same protocol adopted in the present study. The hypothesis of non-specific bands was discarded since a consistent size was observed for all microsatellites across the 103 mango accessions analyzed. This question could only be solved with amplicon gel excision, reamplification, and sequencing of microsatellite alleles differing in size from those reported by Duval et al. (2005) and Schnell et al. (2005), which was not the primary goal of the present study.

The identification of accessions with reference alleles for each microsatellite and their inclusion on the second polyacrylamide gel plate made comparison and correct allelic identification of the remaining accessions possible (Table 3). In the characterization of the grape germplasm bank, Leão et al. (2009) compared the allelic pattern of previous studies and 3 databases of existing microsatellites for the species.

Working with most of the accession analyzed in the present study of the same mango germplasm collection, Santos et al. (2008) reported the formation of 5 groups based on 157 polymorphic AFLP markers: 1) Amrapali, Malika, Embrapa-CPAC hybrids, and some American varieties, 2) predominantly American varieties, with some inclusions of South African and Brazilian hybrids, 3) Brazilian accessions, with some inclusion of Australian, Indian, and American accessions, 4) some accessions of Espada, Rosa, and others of different origins, and 5) Mangifera foetida and M. similis. This AFLP grouping was different from that reported in the present study. Microsatellite markers, which permit the genotyping of individuals, are considered superior to dominant markers such as the AFLP reported by Santos et al. (2008) in mango, and a bigger contribution to the breeding and management of genetic resources for this important crop would be expected with the present study.

The similarity coefficient between 30 and 100\% reflected the high genetic variability of the collection of mango germplasm studied. Santos et al. (2008) reported similarity between 35 and $97 \%$ for the same mango collection with 157 polymorphic AFLP bands. Schnell et al. (1995) analyzed 25 cultivar accessions, mostly from Florida (USA), and found similarity greater than 64\%, while Viruel et al. (2005) observed similarity greater than 33\% in 28 mango accessions from diverse origins, which also demonstrates the high genetic variability of mango.

Gálvez-Lopez et al. (2009) reported high genetic similarity in a study carried out with AFLP and SSR on mango in Mexico, differing from this and previous studies, and which was probably due to constant selection and clonal propagation, which may have reduced genetic diversity. Singh and Bhat (2009) analyzed 18 SSRs in 241 mango genotypes and detected 103 alleles with an average of 5.78 alleles per locus, varying from 3 to 9 alleles per locus. Jaccard's similarity values among the different genotypes varied from 0.024 to 0.808 with an average of 0.258 , indicating the presence of high genetic diversity in the germplasm analyzed. 
Santos et al. (2010) differentiated 44 onion accessions with 13 microsatellite loci and Priolii et al. (2002) differentiated 184 soybean cultivars with the application of only 12 microsatellite SSR markers. According to Hamilton (2009), human forensic DNA profiles use 10-13 unlinked loci to estimate expected genotype frequencies. The microsatellite loci identified in the present study should be sufficient to allow identification of mango cultivars, a crop propagated clonally for commercial production. This study is the first attempt to use microsatellite markers in cultivar protection for the mango agribusiness in Brazil, and can be used to resolve commercial disputes concerning the certification of mango cultivars used in commercial orchards as well.

The present allelic data (Table 3 ) include several major mango cultivars that are grown worldwide, such as the Floridian cultivars, and will be very useful as multiconfirmed reference for identification and protection purposes. However, such a database must be confirmed by other laboratories in order to establish a reliable set of microsatellites to identify mango cultivars, as This et al. (2004) have done with grape cultivars. Suspicious alleles, such as those with larger fragment size, should be discarded and the research should be focused on loci within an expected size range, such as that reported by Duval et al. (2005) and Schnell et al. (2005).

As pointed out by Santos et al. (2010) for onion, laboratories that employ systems that use fluorescent initiators and automatic band recording can estimate different numbers of base pairs for the alleles identified in the present study with the expectation that the allelic pattern is maintained if the same protocol for the PCRs and amplifications is adopted. Studies with DNA codominant markers in mango can be important as references for germplasm management studies, controlled hybridizations, and even for the choice of other pollinators of cultivars that present floral abortion. Mango is an important export crop in many developing countries, and collaborative studies should be carried out in order to define a standard microsatellite set for identification and protection purposes that benefit the entire mango community, especially plant breeders and farmers worldwide.

\section{REFERENCES}

Creste S, Tulmann Neto A and Figueira A (2001). Detection of single sequence repeat polymorphisms in denaturing polyacrylamide sequencing gels by silver staining. Plant Mol. Biol. Rep. 19: 299-306.

Doyle JJ and Doyle JL (1990). Isolation of plant DNA from fresh tissue. Focus 12: 13-15.

Duval MF, Bunel J, Sitbon C and Risterucci M (2005). Development of microsatellite markers for mango (Mangifera indica L.). Mol. Ecol. Notes 5: 824-826.

Gálvez-López D, Hernandéz-Delgado S, Gonzaléz-Paz M, Becerra-Leor EN, et al. (2009). Genetic analysis of mango landraces from Mexico based on molecular markers. Plant Genet. Resour. 7: 244-251.

Hamilton MB (2009). Population Genetics. Wiley-Blackwell, Chichester.

Honsho C, Nishiyama K, Eiadthong W and Yonemori K (2005). Isolation and characterization of new microsatellite markers in mango Mangifera indica. Mol. Ecol. Notes 5: 152-154.

Jakse J, Martin W, Mccallum J and Havey MJ (2005). Single nucleotide polymorphisms, indels, and simple sequence repeats for onion cultivar identification. J. Am. Soc. Hort. Sci. 130: 912-917.

Krishna H and Singh SK (2007). Biotechnological advances in mango (Mangifera indica L.) and their future implication in crop improvement: a review. Biotechnol. Adv. 25: 223-243.

Leão PCS, Riaz S, Graziani R, Dangl GS, et al. (2009). Characterization of a Brazilian grape germplasm collection using microsatellite markers. Am. J. Enol. Vitic. 60: 517-524.

Liu K and Muse SV (2005). PowerMarker: an integrated analysis environment for genetic marker analysis. Bioinformatics 21: 2128-2129.

López-Valenzuela JA, Martínez O and Paredes-López O (1997). Geographic differentiation and embryo type identification in Mangifera indica L. cultivars using RAPD markers. HortScience 32: 1105-1108.

Olano CT, Schnell RJ, Quintanilla WE and Campbell RJ (2005). Pedigree analysis of Florida mango cultivars. Proc. Fla. 
State Hort. Soc. 118: 192-197.

Priolli RHG, Mendes-Junior CT, Arantes NE and Contel EPB (2002). Characterization of Brazilian soybean cultivars using microsatellite markers. Genet. Mol. Biol. 25: 185-193.

Rohlf FJ (1989). NTSYS-pc Numerical Taxonomy and Multivariate Analysis System. Version 1.80. Editora Exeter Software, Setauket.

Santos CAF and Simon PW (2004). Merging carrot linkage groups based on conserved dominant AFLP markers in F2 populations. J. Am. Soc. Hort. Sci. 129: 211-217.

Santos CAF, Lima Neto FP, Rodrigues MA and Costa JG (2008). Similaridade genética de acessos de mangueira de diferentes origens geográficas avaliadas por marcadores AFLP. Rev. Bras. Frutic. 30: 736-740.

Santos CAF, Oliveira VR, Rodrigues MA and Ribeiro HLC (2010). Caracterização molecular de cultivares de cebola com marcadores microssatélites. Pesq. Agropec. Bras. 45: 49-55.

Schnell RJ, Ronning CM and Knight RJ (1995). Identification of cultivars and validation of genetic relationships in Mangifera indica L. using RAPD markers. Theor. Appl. Genet. 90: 269-274.

Schnell RJ, Olano CT, Quintanilla WE and Meerow AW (2005). Isolation and characterization of 15 microsatellite loci from mango (Mangifera indica L.) and cross-species amplification in closely related taxa. Mol. Ecol. Notes 5: 625-627.

Schnell RJ, Brown JS, Olano CT, Meerow AW, et al. (2006). Mango genetic diversity analysis and pedigree inferences for Florida cultivars using microsatellite markers. J. Am. Soc. Hort. Sci. 131: 214-224.

Singh S and Bhat KV (2009). Molecular characterization and analysis of geographical differentiation of Indian mango (Mangifera indica L.) germplasm. Acta Hort. 839: 599-606.

This P, Jung A, Boccacci P, Borrego J, et al. (2004). Development of a standard set of microsatellite reference alleles for identification of grape cultivars. Theor. Appl. Genet. 109: 1448-1458.

Viruel MA, Escribano P, Barbieri M, Ferri M, et al. (2005). Fingerprinting, embryo type and geographic differentiation in mango (Mangifera indica L., Anacardiaceae) with microsatellites. Mol. Breed. 15: 383-393.

Weir BS (1996). Genetic Data Analysis II - Methods for Discrete Population Genetic Data. Sinauer Associates, Sunderland. 